\title{
Compensating Behavior and Constancy of Normalized Energy Transfers in Hall Magnetohydrodynamic Turbulence*)
}

\author{
Keisuke ARAKI and Hideaki MIURA ${ }^{1)}$ \\ Okayama University of Science, Okayama 700-0005, Japan \\ 1) National Institute for Fusion Science, Toki 509-5292, Japan
}

(Received 10 December 2013 / Accepted 7 March 2014)

\begin{abstract}
Using a dissipation-scale adaptive, wavelet-like shell decomposition method and normalization by dissipation-scale characteristics, we found a novel sustaining behavior of self-similarity in the quadratic energy transfer process in freely decaying, fully developed, homogeneous and isotropic turbulences of an incompressible Hall magnetohydrodynamic medium. The process is associated with the relative reduction of nonlinear energy transfer in the dissipation range, which was reported in our previous study [K. Araki and H. Miura, Plasma Fusion Res. 8, 2401137 (2013)]. Gradual reductions in energy transfers by fluid advection and the Hall-term effect are compensated by enhancement of energy transfers due to mutual interactions between velocity and magnetic fields, i.e. between the Lorentz force effect and magnetic induction. This sustaining behavior suggests that coupling between velocity and magnetic fields may be crucial, even when linear dispersive waves aroused by a uniform background magnetic field are absent.
\end{abstract}

(c) 2014 The Japan Society of Plasma Science and Nuclear Fusion Research

Keywords: Hall magnetohydrodynamics, turbulence, energy transfer, wavelet-like shell partitioning method

DOI: $10.1585 /$ pfr.9.3401073

\section{Introduction}

Hall magnetohydrodynamic (HMHD) systems have attracted the interests of many researchers from various viewpoints; for example, the HMHD system has been used as a simple one-fluid magnetohydrodynamic (MHD) model that includes minimal two fluid effects of finite ion skin depth [1] and as a member of "hydrodynamic" mathematical models such as neutral fluids, one-fluid MHD models, and Maxwell-Vlasov systems [2,3].

In the context of nuclear fusion, an appropriate model for multiscale physics simulations is needed to simulate plasma behaviors in large-scale reactors. Large eddy simulations represent one numerical fluid simulation model that has a long history and is still being studied actively [4]. One key to modeling is the understanding of statistical features of turbulent flows; in particular, those of subgridscale motions are essential.

Energy transfer processes in fully developed HMHD turbulence have been studied by direct numerical simulations (DNSs) [5], the closure type approach in the wave turbulence framework [6], mode-truncating coarse-graining approach [7], etc.

Analyzing freely decaying HMHD turbulence allows us to obtain those transfer features that are intrinsic to HMHD systems. By analyzing flow snapshots that exhibit self-similarity, backscatter by the Hall term effect, for example, has been found to occur without forcing [8]. In

author'se-mail: araki@are.ous.ac.jp

*) This article is based on the presentation at the 23rd International Toki Conference (ITC23). our previous study [9], the selective, relatively slow reduction of nonlinear effects was found in dissipation-range dynamics, even after the self-similar energy spectra were established. In the present study, we further investigate this relatively slow variation in the energy transfer process.

\section{Basic Equations, Parameters, and Analyzed Fields}

In an HMHD system, the velocity field $\boldsymbol{u}$ and magnetic field $\boldsymbol{b}$ in normalized units obey the following equations:

$$
\begin{aligned}
& \partial_{t} \boldsymbol{u}+(\boldsymbol{u} \cdot \nabla) \boldsymbol{u}=\nabla P+\boldsymbol{j} \times \boldsymbol{b}+v \Delta \boldsymbol{u}, \\
& \partial_{t} \boldsymbol{b}=\nabla \times((\boldsymbol{u}-\alpha \boldsymbol{j}) \times \boldsymbol{b})+\eta \Delta \boldsymbol{b}, \\
& \nabla \cdot \boldsymbol{u}=\nabla \cdot \boldsymbol{b}=0,
\end{aligned}
$$

where $P, \boldsymbol{j}:=\nabla \times \boldsymbol{b}, \nu, \alpha$ and $\eta$ are generalized pressure, current density field, kinematic viscosity, the parameter specifying the relative strength of the Hall term effect, and resistivity, respectively.

DNSs were carried out using the 2/3-dealiasing pseudospectral method with $N=512^{3}$ grid points. The numerical schemes are detailed in [10]. For the present study, the parameters were set to $v=\eta=1 \times 10^{-3}$ and $\alpha=0.05$. No external force was imposed on the fields.

Analyzed snapshot datasets were taken from two DNS runs, which we hereafter call Runs A and B. The runs were carried out with the same parameters given above but with different initial conditions that had the same kinetic and magnetic energies with the same energy spectra given in [10] but different magnetic and hybrid helicities. 

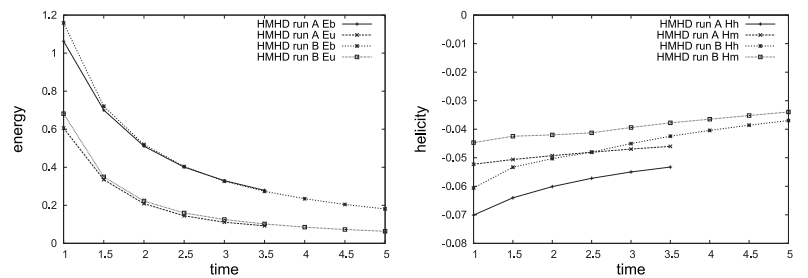

Fig. 1 Time development of energies and helicities in Runs A and B. Left: kinetic energy $E_{u}$ and magnetic energy $E_{b}$; right: magnetic helicity $H_{m}:=\int \boldsymbol{a} \cdot \boldsymbol{b} \mathrm{d}^{3} \vec{x}$ and hybrid helicity $H_{h}:=\int(\alpha \boldsymbol{u}+\boldsymbol{a}) \cdot(\alpha \nabla \times \boldsymbol{u}+\boldsymbol{b}) \mathrm{d}^{3} \vec{x}$, where $\boldsymbol{a}$ is vector potential.

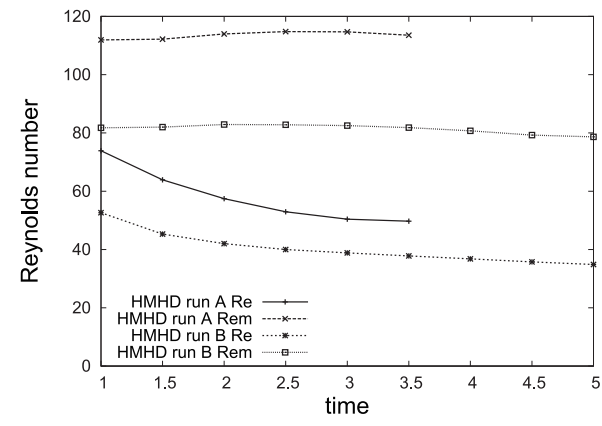

Fig. 2 Time development of the Taylor microscale Reynolds number $\operatorname{Re}:=\sqrt{10 / 3 v \epsilon_{K}} E_{u}$, and its magnetic counterpart Rem $:=\sqrt{10 / 3 \eta \epsilon_{B}} E_{b}$, where $\epsilon_{K}, \epsilon_{B}$ are dissipation rates of kinetic and magnetic energies.

Six snapshot datasets $(t=1.0,1.5, \ldots, 3.5)$ were taken from Run A, which were analyzed in $[8,11]$. Nine snapshot datasets $(t=1.0,1.5, \ldots, 5.0)$ were taken from Run B, which were analyzed in [9]. Both snapshot datasets were collected after turbulence had developed sufficiently.

During the analyzed time period, the two runs showed very close kinetic and magnetic energies (see Fig. 1, left). For magnetic and hybrid helicities, which are well known to be constants of the motion in the dissipationless limit of HMHD, the Run A solution had moduli approximately 15\% larger than Run B (see Fig. 1, right). But both runs had good constancy in the magnetic Reynolds number (see Fig. 2) and self-similarity in the magnetic and kinetic energy spectra $[8,9]$.

\section{Dissipation-Scale Adaptive, Wavelet-Like Shell Decomposition Method}

For this study, we used the dissipation-scale adaptive, dyadic wavelet like, shell decomposition method that was introduced in our previous study [9]. Wavenumber space was decomposed into spherical shells with the $j$-th shell occupying the wavenumber range given by $2^{-(j+1) / 2} k_{\eta}<$ $k<2^{-j / 2} k_{\eta}$, where $k_{\eta}:=\sqrt[4]{\epsilon_{B}(t) / \eta^{3}}$ is the characteristic wavenumber of the dissipation range and $\epsilon_{B}(t):=\eta \int \mid \nabla \times$ $\left.\boldsymbol{b}(\vec{x}, t)\right|^{2} \mathrm{~d}^{3} \vec{x}$ is the dissipation rate of the magnetic field at time $t$. Each field was decomposed into band-pass-filtered components as

$$
f(\vec{x}, t)=\sum_{j} f_{j}(\vec{x}, t)
$$

where $f$ stands for the velocity and magnetic fields. Note that the shell index $j$, which denotes the characteristic motion scale of the corresponding field, is $\sqrt{2^{j}}$ times the Kolmogorov scale of the magnetic field.

Since here we are analyzing freely decaying turbulence, the wavenumber of the Kolmogorov scale gradually decreases over time. Therefore, the width of the wavenumber range for the $j$-th shell also gradually decreases over time; this corresponds to dissipation of small-scale motions and enlargement of coherent structures such as vortex tubes and current sheets.

Decomposing the basic equations (1)-(3) and taking inner products with the $j$-th mode, we obtain the energy budget equations for velocity and magnetic fields as follows:

$$
\begin{aligned}
& \frac{\mathrm{d}}{\mathrm{dt}} E_{j}^{(u)}=\sum_{k, m}\left\langle\boldsymbol{u}_{j}\left|\boldsymbol{u}_{m}\right| \boldsymbol{u}_{k}\right\rangle_{a d v}+\sum_{k, m}\left\langle\boldsymbol{u}_{j}\left|\boldsymbol{b}_{m}\right| \boldsymbol{b}_{k}\right\rangle_{\text {Lor }}+D_{j}, \\
& \frac{\mathrm{d}}{\mathrm{dt}} E_{j}^{(b)}=\sum_{j, m}\left\langle\boldsymbol{b}_{j}\left|\boldsymbol{b}_{m}\right| \boldsymbol{u}_{k}\right\rangle_{\text {Ind }}+\sum_{j, m}\left\langle\boldsymbol{b}_{j}\left|\boldsymbol{b}_{m}\right| \boldsymbol{b}_{k}\right\rangle_{\text {Hall }}+R_{j},
\end{aligned}
$$

where the quadratic interaction terms are defined by

$$
\begin{aligned}
\left\langle\boldsymbol{u}_{j}\left|\boldsymbol{u}_{m}\right| \boldsymbol{u}_{k}\right\rangle_{a d v} & :=-\int\left(\left(\boldsymbol{u}_{m} \cdot \nabla\right) \boldsymbol{u}_{k}\right) \cdot \boldsymbol{u}_{j} \mathrm{~d}^{3} \vec{x}, \\
\left\langle\boldsymbol{u}_{j}\left|\boldsymbol{b}_{m}\right| \boldsymbol{b}_{k}\right\rangle_{\text {Lor }} & :=\int\left(\boldsymbol{j}_{k} \times \boldsymbol{b}_{m}\right) \cdot \boldsymbol{u}_{j} \mathrm{~d}^{3} \vec{x}, \\
\left\langle\boldsymbol{b}_{j}\left|\boldsymbol{b}_{m}\right| \boldsymbol{u}_{k}\right\rangle_{\text {Ind }} & :=\int\left(\nabla \times\left(\boldsymbol{u}_{k} \times \boldsymbol{b}_{m}\right)\right) \cdot \boldsymbol{b}_{j} \mathrm{~d}^{3} \vec{x}, \\
\left\langle\boldsymbol{b}_{j}\left|\boldsymbol{b}_{m}\right| \boldsymbol{b}_{k}\right\rangle_{\text {Hall }} & :=-\alpha \int\left(\nabla \times\left(\boldsymbol{j}_{k} \times \boldsymbol{b}_{m}\right)\right) \cdot \boldsymbol{b}_{j} \mathrm{~d}^{3} \vec{x} .
\end{aligned}
$$

Definitions of the other terms and a discussion of the choice of integrands are given in $[9,12]$. In the following section, we sum them up with respect to $k$ and $m$ and denote the sums as $\left\langle\boldsymbol{u}_{j}|\boldsymbol{u}| \boldsymbol{u}\right\rangle_{a d v}:=\sum_{k, m}\left\langle\boldsymbol{u}_{j}\left|\boldsymbol{u}_{m}\right| \boldsymbol{u}_{k}\right\rangle_{a d v}$, and so on.

\section{Numerical Result}

Figure 3 shows normalized net quadratic transfer functions for the kinetic and magnetic energies. As discussed in [9], normalization by the dissipation-scale characteristics, $\epsilon_{B}(t)$ and $\eta$, renders the amplitudes of the energy transfer functions comparable between different time snapshots. Thus, we expect that the principal differences at different times are due to topological features of their vector fields of each run.

The panels in Fig. 3 show that the functional profiles of the total kinetic and magnetic energy transfers tend to converge, over time, to a single profile. This convergence occurs for wavenumber-space shell numbers $j \leq 4\left(k / k_{\eta}>\right.$ $0.2)$ for kinetic energy and $2 \leq j \leq 6\left(0.1<k / k_{\eta}<0.4\right)$ for 

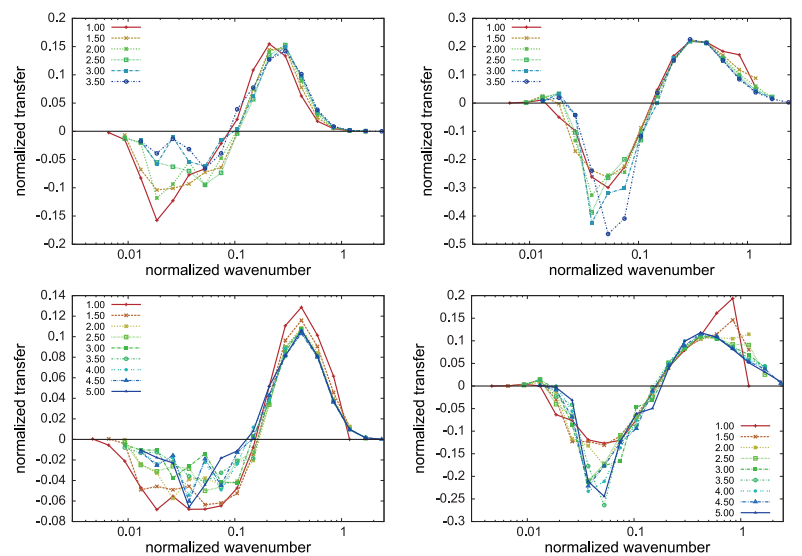

Fig. 3 Time development of total energy transfer functions for kinetic and magnetic energies. Left: kinetic energy, $\left\langle\boldsymbol{u}_{j}|\boldsymbol{u}| \boldsymbol{u}\right\rangle_{a d v}+\left\langle\boldsymbol{u}_{j}|\boldsymbol{b}| \boldsymbol{b}\right\rangle_{\text {Lor }}$; right: magnetic energy $\left\langle\boldsymbol{b}_{j}|\boldsymbol{b}| \boldsymbol{u}\right\rangle_{\text {Ind }}+\left\langle\boldsymbol{b}_{j}|\boldsymbol{b}| \boldsymbol{b}\right\rangle_{\text {Hall }}$. Top: Run A; bottom: Run B. Changes in line colors from red to blue denote time development of the transfer functions. Abscissa of $j$-th shell data is set to $k_{j}=\sqrt{2^{-j-1 / 2}} k_{\eta}$.
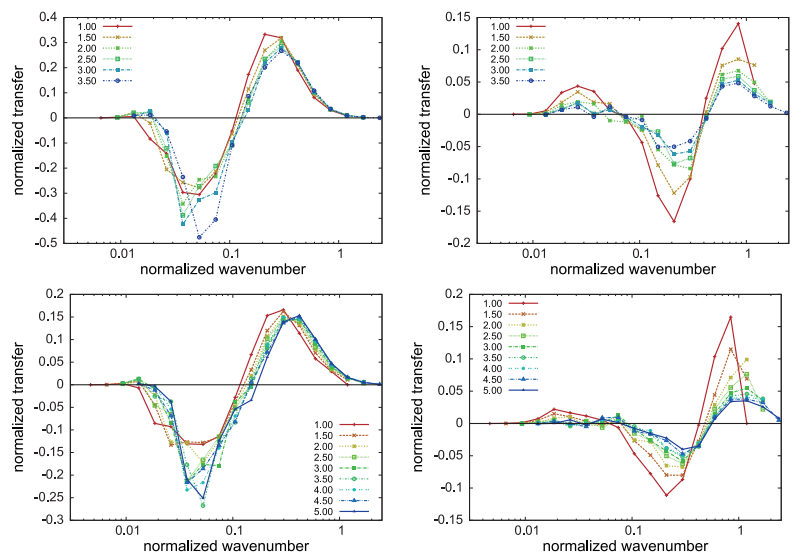

Fig. 4 Time development of magnetic energy transfers due to each term of HMHD equations. Left: magnetic induction effect $\left\langle\boldsymbol{b}_{j}|\boldsymbol{b}| \boldsymbol{u}\right\rangle_{\text {Ind }}$; right: Hall-term effect $\left\langle\boldsymbol{b}_{j}|\boldsymbol{b}| \boldsymbol{b}\right\rangle_{\text {Hall }}$. Top: run A; bottom: run B. Meaning of the line colors are same as Fig. 3.

magnetic energy. The magnetic energy transfer function also shows a tendency to converge to a single profile for smaller motion scales $j \leq 1\left(k / k_{\eta}>0.4\right)$.

It is remarkable that this stationarity of normalized energy transfer is not caused by constancy of transfer of individual quadratic terms, but by compensating switching between them.

In Fig. 4, we separately show the time development in magnetic energy transfer due to magnetic induction $\left\langle\boldsymbol{b}_{j}|\boldsymbol{b}| \boldsymbol{u}\right\rangle_{\text {Ind }}$ and the Hall-term effect $\left\langle\boldsymbol{b}_{k}|\boldsymbol{b}| \boldsymbol{b}\right\rangle_{\text {Hall }}$.

For example, for the shell having $j=4\left(k / k_{\eta} \sim 0.2\right)$, the modulus of nonlinear transfer by the Hall-term effect gradually decreases over time, even after the quantities are normalized by the dissipation rate. The modulus of the
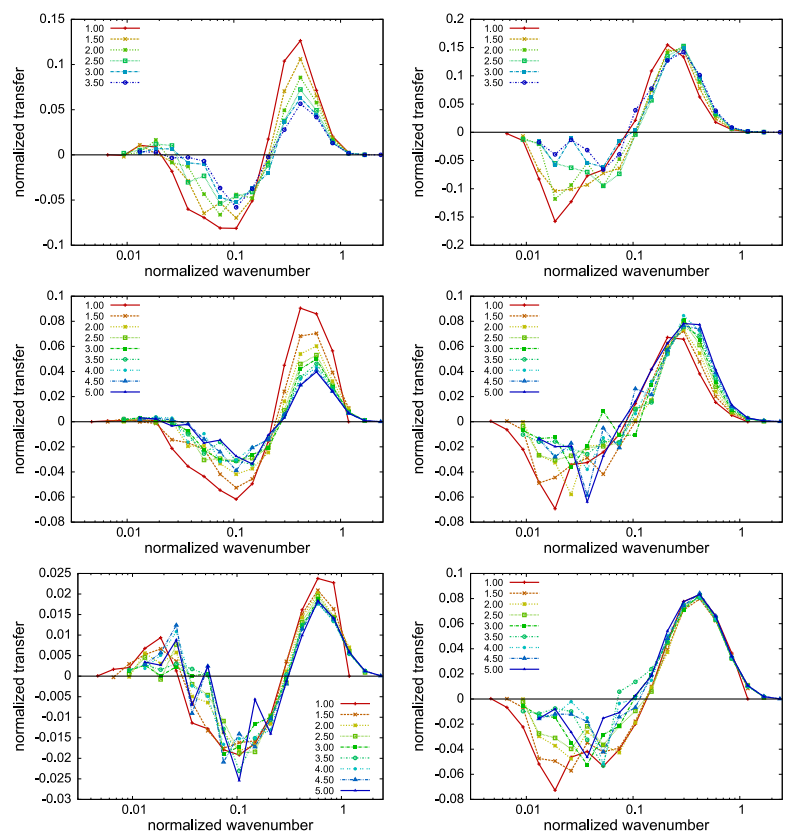

Fig. 5 Time development of kinetic energy transfers due to each term in HMHD equations. Left: fluid advection effect $\left\langle\boldsymbol{u}_{j}|\boldsymbol{u}| \boldsymbol{u}\right\rangle_{a d v}$; right: Lorentz force effect $\left\langle\boldsymbol{u}_{j}|\boldsymbol{b}| \boldsymbol{b}\right\rangle_{L o r}$. Top: Run A; middle: Run B; bottom: MHD run with the same dissipation coefficients and initial conditions as Run B [9]. Meaning of line colors are same as in Fig. 3.

magnetic induction effect also decreases over time, while the positive peak values in the induction effect are almost constant $\left(\left\langle\boldsymbol{b}_{j}|\boldsymbol{b}| \boldsymbol{u}\right\rangle_{\text {Ind }} \sim 2.8\right.$ for Run A, 1.5 for Run B). Despite these decreases, the net transfer shows remarkable constancy.

As another example, for the shell with $j=1\left(k / k_{\eta} \sim\right.$ $0.6)$, reduction in the Hall-term effect is compensated by enhancement of induction effect, therefore, the net transfer is also nearly constant for $t \geq 1.5$.

Figure 5 shows the time development of kinetic energy transfer due to fluid advection and the Lorentz force effect. Note that the peak values of fluid advection energy transfer occur at somewhat higher wavenumbers than those in the Lorentz force effect.

Noticeable reduction in the fluid advection effect and compensation by the Lorentz force effect occur in the wavenumber shell number $j<2\left(k>0.4 k_{\eta}\right)$, especially for Run B.

\section{Discussion}

In the present study, we found constancy in the net quadratic energy transfer of kinetic and magnetic energies and compensating switching between the effects of quadratic terms. These compensating effects occur in dissipation scale motions whose characteristic wavenumbers have $k>0.1 k_{\eta}$; these scale motions are smaller than about 10 times the Kolmogorov scale. As is discussed in [9], such slow relaxation phenomena are not found in MHD 
systems (see Fig. 5), which suggests that they may be intrinsic to HMHD systems.

Our finding suggests that the energy transfer due to velocity and magnetic fields may not independently occur but in some coupled manner. One possible explanation for this coupling is that interactions occurs via coupling of linear eigenmodes, i.e., ion cyclotron modes and whistler modes of the HMHD system

It is well known that, when an external uniform magnetic field (say $\boldsymbol{B}_{0}$ ) is imposed, a linearized system of the basic equations (1) and (2) gives a dispersion relation whose eigenfrequencies are given by $\omega_{s}(\vec{k}, \sigma)=$ $\lambda_{s}(\vec{k}, \sigma) \boldsymbol{B}_{0} \cdot \vec{k}$ (for example, see [6]). Here, the $\lambda$ 's are defined by

$$
\lambda_{s}(\vec{k}, \sigma):=\frac{1}{2}\left(-\sigma \alpha|\vec{k}|+s \sqrt{(\alpha|\vec{k}|)^{2}+4}\right),
$$

where $s= \pm 1$ and $\sigma= \pm 1$ are the signs that defines the eigenmode branches and polarity of the polarized Fourier mode, respectively. The associated eigenvectors are given by

$$
\left(\begin{array}{c}
\boldsymbol{u} \\
\boldsymbol{b}
\end{array}\right)=\frac{\hat{u}+\lambda_{s} \hat{b}}{\lambda_{s}^{2}+1}\left(\begin{array}{c}
\boldsymbol{\phi}(\vec{k}, \sigma ; \vec{x}) \\
\lambda_{s} \boldsymbol{\phi}(\vec{k}, \sigma ; \vec{x})
\end{array}\right),
$$

where $\phi(\vec{k}, \sigma ; \vec{x})$ is a complex helical wave (CHW) vector field (see [4], Sect. V-5) with wavenumber $\vec{k}$ and polarity $\sigma$, while $\hat{u}$ and $\hat{b}$ are the Fourier coefficients of the velocity and magnetic fields with respect to $\mathrm{CHW}$. It is interesting that, since the $\lambda$ 's in equation (11) do not include $\boldsymbol{B}_{0}$ as a parameter, the decomposition to these eigenmodes is applicable to systems without external uniform magnetic fields.

The moduli of the eigen frequencies are $\left|\omega_{s}\left(\vec{k}, \sigma_{k}\right)\right|=$
$\left|\boldsymbol{B}_{0} \cdot \vec{k}\right| \lambda$ and $\left|\boldsymbol{B}_{0} \cdot \vec{k}\right| / \lambda$, where $\lambda=\frac{1}{2}\left(\alpha|\vec{k}|+\sqrt{(\alpha|\vec{k}|)^{2}+4}\right)$, and correspond to whistler and ion cyclotron waves, respectively. In the present study, we imposed no such external magnetic field $\boldsymbol{B}_{0}$, but large scale structures of the magnetic field may surrogate $\boldsymbol{B}_{0}$. The split to these rapid and slow modes may explain the slow relaxation of quadratic interaction effects, and an analysis is now underway.

This work was performed under the auspices of the NIFS Collaboration Research Program (NIFS13KNSS044) and KAKENHI (Grant-in-Aid for Scientific Research(C)) 23540583.

[1] M.J. Lighthill, Phil. Trans. Roy. Soc. London 252A, 397 (1960).

[2] J.E. Marsden and T.S. Ratiu, Introduction to Mechanics And Symmetry: Second Edition (Springer, New York, 1999).

[3] K. Araki, J. Math-for-Industry 1, 139 (2009).

[4] M. Lesieur, Turbulence in Fluids: Third Revised and Enlarged Edition (Kluwer, Dortrecht, 1997).

[5] P.D. Mininni, A. Alexakis and A. Pouquet, J. Plasma Phys. 73, 377 (2007).

[6] S. Galtier, J. Plasma Phys. 72, 721 (2006).

[7] H. Miura and K. Araki, Plasma Phys. Control. Fusion 55, 014012 (2013).

[8] K. Araki and H. Miura, Plasma Fusion Res. 6, 2401132 (2011).

[9] K. Araki and H. Miura, Plasma Fusion Res. 8, 2401137 (2013).

[10] H. Miura and D. Hori, J. Plasma Fusion Res. SERIES 8, 73 (2009).

[11] K. Araki and H. Miura, Plasma Fusion Res. 5, S2048 (2010).

[12] K. Araki and H. Miura, J. Plasma Fusion Res. SERIES 9, 446 (2010). 